\title{
Health and income: A robust comparison of Canada and the US
}

\author{
Jean-Yves Duclos*and Damien Échevin ${ }^{\dagger}$
}

April 20, 2009

\begin{abstract}
This paper uses sequential stochastic dominance procedures to compare the joint distribution of health and income across space and time. It is the first application of which we are aware of methods to compare multidimensional distributions of income and health using procedures that are robust to aggregation techniques. The paper's approach is more general than comparisons of health gradients and does not require the estimation of health equivalent incomes. We illustrate the approach by contrasting Canada and the US using comparable data. Canada dominates the US over the lower bi-dimensional welfare distribution of health and income, though not generally in terms of the uni-dimensional distribution of health or income. The paper also finds that welfare for both Canadians and Americans has not unambiguously improved during the last decade over the joint distribution of income and health, in spite of the fact that the uni-dimensional distributions of income have clearly improved during that period.
\end{abstract}

Keywords: Health inequality; Self-reported health status; Income distribution; Stochastic dominance; Social welfare.

JEL Numbers: I10; I32; I38; D63; D30; H51.

* Institut d'Anàlisi Econòmica (CSIC), Barcelona, Spain, and Département d'économique and CIRPÉE, Université Laval, Canada; email: jyves@ecn.ulaval.ca

†Département d'économique and GRÉDI, Université de Sherbrooke, 2500 bd de l'Université, Sherbrooke (Québec), J1K 2R1, Canada; e-mail: damien.echevin@usherbrooke.ca; fax: +1 819821-7934; phone: +1 819-821-8000 (ext. 61358). 


\section{Introduction}

The recent literature on health economics has shown a keen interest in the measurement of health inequality and in its determinants. One of the more persistent and salient findings ${ }^{1}$ is the existence of a "health-income gradient", in the sense that health tends to be associated positively with incomes. A seemingly natural corollary is that health policy might usefully take place through income redistribution and a general improvement in living standards.

A number of important difficulties arise, however, when it comes to analyzing (and using) the link between income and health for descriptive and policy purposes. Trying to influence health through impacting on endogenous socioeconomic variables such as income is tricky because of possible dual causality income may affect health, but health may also affect income, or at least be correlated with it through other channels. Health-income links are also heterogeneous, in part because health-related risk behavior is diversified and depends inter alia on where individuals are placed in the income distribution ${ }^{2}$.

The measurement of health inequality also faces the important problem that most available health indicators are of a qualitative nature, or at least cannot be considered to have a cardinal content (such as for the usual self-reported subjective health indicators). This makes it difficult to use such indicators with conventional measures of inequality, in part because conventional measures of relative inequality need to use distributional means and that these are difficult to interpret when variables of well-being are not cardinal ${ }^{3}$.

In spite of these difficulties, the analysis of the joint distribution of income and health is important in order to apprehend well-being, partly because well-being is increasingly considered to be multidimensional in the first place. The current paper contributes to that literature by proceeding to a two-dimensional analysis of well-being that differs from previous work in three different ways: 1) it is more

\footnotetext{
${ }^{1}$ See for instance Cutler, Deaton, and Lleras-Muney (2006), Marmot (2002), O'Neill and O'Neill (2007), and Deaton (2003).

${ }^{2}$ In the presence of such heterogeneity, Contoyannis and Forster (1999) show for instance that a public policy that reduces income inequality may, under certain circumstances, leave health inequality unchanged or even raise it.

${ }^{3} \mathrm{~A}$ variety of methods have been proposed to re-scale ordinal measures of health into cardinal ones - for examples and a discussion of the procedures, see Wagstaff, Paci, and van Doorslaer (1991), Wagstaff and van Doorslaer (1994), Deaton and Paxson (1998), Humphries and van Doorslaer (2000), Wagstaff and van Doorslaer (2000), van Doorslaer and Jones (2003), Allison and Foster (2004), and Zheng (2006).
} 
general than an analysis based on health gradients; 2) it is not hampered by the ordinal nature of most health indicators; 3 ) and it is more robust to aggregation techniques than the aggregation procedures usually found in the literature.

In practice, empirical work on multidimensional indicators of well-being juxtaposes several indicators of well-being either by studying the uni-dimensional (also called the marginal) distributions of each independently or, alternatively, by aggregating somewhat arbitrarily the set of well-being indicators into a single one. In order to compare well-being using both income and health indicators, this paper proceeds instead by using two sets of relatively weak measurement conditions that are sequentially applied to the measurement system. The first set of conditions is that (i) both income and health are assumed to generate well-being (better health can make up for lesser income, and conversely), and that (ii) the well-being impact of an increase in income decreases with health (greater income increases well-being, but the more so for the less healthy). The second set of conditions is that (iii) well-being is concave in incomes (transferring income from a better-off individual to a poorer one raises social welfare - this is also called the PigouDalton principle of transfer), and that (iv) the income concavity of social welfare decreases with health levels (to improve social welfare, a transfer involving people that are both poor and sick will have a greater impact than one aimed at poor but healthy people).

The paper then uses ranking techniques based on multidimensional stochastic dominance ${ }^{4}$. The techniques can be applied to any bidimensional ranking of wellbeing. It does not need to assume a particular functional relationship between the two variables - in particular, it does not presume that income and health are positively associated. As mentioned above, it is also not hampered by the ordinal nature of a health indicator, and it also uses a more general aggregation technique than most of the procedures found in the literature.

Applying that approach enables us to compare the joint health and income distributions of Canada and of the US. This is first done using the Joint Canada/United States Survey of Health. It is well known that the two countries have a very different health care system. Canada's health care system is almost entirely public and is regulated by national rules that purport to enforce universality of access and of quality. The US health care system is more diverse, with a more significant mixture of public and private provision and financing that is

\footnotetext{
${ }^{4}$ One of the first applications of this approach has been in the context of welfare comparisons of households with heterogenous sizes and compositions - see for instance Atkinson and Bourguignon (1982) and Atkinson (1992) for early contributions.
} 
conducive to greater freedom of choice and competition, but at the possible cost of greater heterogeneity in access to and quality of care. As we will see (and as is also well known), the income distributions also differ significantly across the two countries, with the US displaying both greater average incomes and greater income inequality than Canada. Thus, not only can we expect the uni-dimensional distributions of incomes and health distributions to differ across the two countries, but we can also expect the gradient and the correlation between these distributions to be different.

We also use two national surveys, the Canadian National Population Health Survey (recently changed to the Canadian Community Health Survey) and the US National Health Interview Survey, in order to obtain longitudinal comparisons of the two countries in a context of important and rapid changes in the distributions of both incomes and health statuses in the two countries.

The rest of the paper is structured as follows. Section 2 describes the sequential stochastic dominance approach. Section 3 presents the results of the comparisons between the US and Canada along with the results of the comparisons across time of both countries. Section 4 summarizes the results.

\section{Joint welfare dominance}

Suppose that the population is split into $K$ groups of health statuses with population share denoted by $\phi(k), k=1, \ldots, K$. Hence $\sum_{k=1}^{K} \phi(k)=1$. Denote $h_{k}$ the (ordinal or cardinal) value of the health level of those in group $k, k=1, \ldots, K$. Health statuses are ranked in increasing order from the lowest to the greatest health status, i.e., $h_{1} \leq h_{2} \leq \ldots \leq h_{K}$.

Define $F(y ; k)$ as the income distribution function of those in health group $k$ - it shows the proportion of individuals in group $k$ that have incomes less than or equal to $y$. We will use the vector $(z(1), \ldots, z(K))$ to allow us to focus (if we so choose) on different portions of the income distribution, $y \leq z(k)$, depending on the health group. Furthermore, define $\omega_{k}(y)$ as the contribution to social welfare of an individual with income $y$ that belongs to group $k$. Note that $\omega_{k}(y)$ will implicitly depend on $z(k)$. Aggregating across incomes and across groups, this can be used to obtain a general measure $W$ of social welfare jointly over health and income:

$$
W(z(1), \ldots, z(K))=\sum_{k=1}^{K} \phi(k) \int_{0}^{\infty} \omega_{k}(y) d F(y ; k) .
$$


We assume that the social welfare function is such that

$$
\begin{array}{r}
z(1) \geq z(2) \geq \ldots \geq z(K), \\
\omega_{1}^{(1)}(y) \geq \ldots \geq \omega_{K}^{(1)}(y) \geq 0, \forall y, \\
\omega_{k}(y)=c, \forall k=1, \ldots, K \text { and } \forall y \geq z(k)
\end{array}
$$

where $\omega_{k}^{(s)}(y)$ is the $s$-order derivative of $\omega_{k}(y)$ with respect to $y$.

Assumption (2) means that, for measuring social welfare, the income threshold $z(k)$ for a given health group $k$ cannot exceed the threshold set for less healthy individuals since presumably these less healthy individuals have higher needs (in terms of use of health care for instance) than healthier individuals. Note that $z(k)$ does not have to be upper bounded. It can in particular be set to exceed the maximum income in a group $k$, subject to (2).

Assumption (3) says that income $y$ contributes to the production of well-being (the first-order derivative of $\omega$ is positive). An increase in income is always good for well-being and social welfare. Such an increase is, however, particularly good for those with a worse health status, as implied by the ordering of the first-order derivatives in (3). This implicitly allows one to be concerned about the joint distribution of income and health. For given marginal distributions of income and health, an increase in the correlation between income and health is equivalent to thinking about a redistribution of income from those with lower health to those with higher health. This will decrease welfare according to (3).

The combination of assumptions (2) and (4) implicity says that health also contributes to well-being. The reason is that it takes a lower income $z(k)$ (see (2)) at a higher health level to generate the same level of well-being $c$ in (4). Combining this with (3), we can also show that well-being $\omega_{k+1}(y)$ for a member of a group $k+1$ is always larger than well-being $\omega_{k}(y)$ for a member of a group $k$ at any common value of $y$. This therefore says that those with a better health status achieves a greater level of well-being at any given common income level.

Assumption (4) also allows to focus only on those with the lowest levels of well-being. This is why it is assumed that $\omega_{k}(y)=c, \forall k=1, \ldots, K$ and $\forall y \geq$ $z(k)$; as we will see, however, the methodology is general enough to allow for wide ranges of values for $z(k)$. This leads to the following first main result.

Theorem 1 (First-order welfare dominance) Consider two joint distributions $A$ and $B$ of income and health and define $\Delta F(y ; k)=\phi_{A}(k) F_{A}(y ; k)-$ $\phi_{B}(k) F_{B}(y ; k)$. Then, social welfare over the distribution of health and income is 
higher in population A than in population $B$ for all of the social welfare functions $W(z(1), \ldots, z(K))$ in (1) that obey assumptions (2), (3) and (4) if and only if

$$
\sum_{k=1}^{i} \Delta F(y ; k)<0, \forall y<z(i), \forall i=1, \ldots, K
$$

Proof. See the Appendix.

Condition (5) is analogous to the sequential dominance condition in the social welfare literature with heterogeneous agents - see Atkinson and Bourguignon (1987) and Jenkins and Lambert (1993) for instance. It says that we should first compare the income distribution of the group of the least healthy, then of the two least healthy groups, and so on, until the entire income distributions are eventually compared (up to the lowest $z(i)$ ). Equivalently, condition (5) compares the joint distribution of both income and health, and implies that $A$ has more welfare than $B$ if and only if the proportion of people that have incomes below $y$ and health status below or at $h_{k}$ is always lower in $A$. This also says that although the marginal distributions (obtained in (5) when $i=K$ or when $y$ tends to infinity) do matter, the correlation between the dimensions also matters in establishing dominance.

Theorem 1 is a very general result that allows for a wide range of assumptions on the interaction of health and income in the evaluation of individual well-being (the function $\omega_{k}(y)$ ) and social welfare (the function $W$ ). It also allows for a wide range of possible values for the vector $(z(1), \ldots, z(K))$. In fact, if (5) holds, then it is simple to show that the ranking of $A$ and $B$ provided by Theorem 1 is even wider.

Corollary 1 Consider two joint distributions $A$ and $B$ of income and health and define $\Delta F(y ; k)=\phi_{A}(k) F_{A}(y ; k)-\phi_{B}(k) F_{B}(y ; k)$. Then, social welfare over the distribution of health and income is higher in population A than in population $B$ for all of the social welfare functions $W(\zeta(1), \ldots, \zeta(K))$ in $(1)$ that obey assumptions (2), (3) and (4) and for all values of $\zeta(k) \leq z(k), k=1, \ldots, K$, if and only if

$$
\sum_{k=1}^{i} \Delta F(y ; k)<0, \forall y<z(i), \forall i=1, \ldots, K .
$$

Proof. The proof follows from the proof of Theorem 1. 
The second main result is obtained through making additional assumptions on the functions $\omega$. Let

$$
\omega_{1}^{(2)}(y) \leq \ldots \leq \omega_{K}^{(2)}(y) \leq 0, \forall y
$$

Assumption (7) says that the second derivative of $\omega_{k}(y)$ should be negative. Social welfare indices are then concave in $y$ and are thus increasing in meanpreserving equalizing transfers of income; that is, they should obey the PigouDalton principle of transfers (within each group $k$ ) and increase if a dollar of income is redistributed from a richer to a poorer person. The distribution of income within a group is therefore important in order to assess the contribution of that group to social welfare. In assumption (7), the concavity of $\omega_{k}(y)$, and thus the social welfare importance of the Pigou-Dalton principle of transfers, is also assumed to be decreasing in $k$ and hence increasing in the needs of the groups. Said differently, the lower the health level of that group, the greater the welfare cost of inequality in that group.

Let

$$
D(z ; k)=\int_{0}^{z}(z-y) d F(y ; k) .
$$

In the poverty literature, (8) is referred to as the average "poverty gap" for group $k$ at poverty line $z$. The second main result follows.

Theorem 2 (Second-order welfare dominance) Consider two distributions of income and health $A$ and $B$ and define $\Delta D(y ; k)=\phi_{A}(k) D_{A}(y ; k)-$ $\phi_{B}(k) D_{B}(y ; k)$. Social welfare is then higher in population $A$ than in population $B$ for all of the social welfare functions $W(z(1), \ldots, z(K))$ in $(1)$ that obey assumptions (2), (3), (4) and (7) if and only if

$$
\sum_{k=1}^{i} \Delta D(y ; k)<0, \forall y<z(i), \forall i=1, \ldots, K .
$$

Proof. As for the proof of Theorem 1.

Condition (9) is identical to (5) except that it compares average income gaps (instead of cumulative distribution functions) across distributions. Income gaps are sensitive to the depth of income deprivation, unlike the distribution functions used in (5). This is the reason for which Theorem (2) can rank social welfare functions that are sensitive to income inequality. Societies with less income inequality 
are less likely to lead to higher incomes gaps within health groups, and are thus more likely to be considered better by criterion (9). The scope of Theorem (2) can be extended as for Theorem (1):

Corollary 2 Consider two distributions of income and health $A$ and $B$ and define $\Delta D(y ; k)=\phi_{A}(k) D_{A}(y ; k)-\phi_{B}(k) D_{B}(y ; k)$. Then, social welfare over the distribution of health and income is higher in population $A$ than in population $B$ for all of the social welfare functions $W(\zeta(1), \ldots, \zeta(K))$ in (1) that obey assumptions (2), (3), (4) and (7) and for all values of $\zeta(k) \leq z(k), k=1, \ldots, K$, if and only if

$$
\sum_{k=1}^{i} \Delta D(y ; k)<0, \forall y<z(i), \forall i=1, \ldots, K .
$$

Proof. As above.

\section{Application: comparing Canada and the US}

We now use the tools introduced above to compare well-being between Canada and the US using the 2003 Joint Canada/United States Survey of Health (JCUSH). This survey provides detailed information on income (a continuous variable ${ }^{5}$ ) and health (qualitative self-reported health status). One important advantage of using this survey is that the same questionnaire was used in both countries, thus leading to exactly the same definition of the variables. One limit of the survey, however, is that income in both countries is after transfers but before taxes.

We apply purchasing power parities from the OECD to convert the Canadian currency into 2003 US dollars (that is, 1 \$US = 1.23 \$CA in 2003). Furthermore, as is often done in the literature, we divide total household income by an adult-equivalence scale defined as $h^{0.5}$, where $h$ is household size. Incomes below $\$ 5,000$ are set to $\$ 5,000$, and those above $\$ 100,000$ (or $\$ 120,000$ ) are censored at $\$ 100,000$ (or $\$ 120,000$ ).

\footnotetext{
${ }^{5}$ Note that the income variable is not continuous for those households that declared it through a system of eleven intervals. A simple econometric model has thus been used in order to predict income for those households and then simulate it within the intervals. Details of this procedure are available on request from the authors.
} 


\subsection{Canada-US comparisons}

Figure 1 plots the cumulative distribution functions of the Canadian and American income distributions ${ }^{6}$. Canada dominates the US over relatively low income thresholds, viz, income headcounts in the US are higher than in Canada when income thresholds are chosen to be below approximately $\$ 10,000$. For higher thresholds, however, headcounts are clearly larger in Canada, and US incomes are therefore larger than those in Canada over a wide range of percentiles. Table 1 presents the cumulative frequencies of health statuses in each country. No country seems to dominate strongly the other in terms of health. The overall ranking of the two countries in terms of both income and health would therefore also seem to be ambiguous.

We thus proceed to check for social welfare dominance jointly in both dimensions. Table 2 presents $95 \%$ asymptotically derived confidence intervals for the estimates of $\sum_{k=1}^{i} \Delta F(z ; k)$ and $\sum_{k=1}^{i} \Delta D(z ; k)$ for different cumulative values of $i$ (that is for the different health statuses ranked in ascending order, from poor health to excellent health) and for various income thresholds $z$. The point estimates can be read as the midpoint of the confidence intervals (since these are symmetrically spread around the point estimates).

We begin by checking for first-order dominance. This is done in Table 2 by considering the estimates and the intervals under the columns headed by "(1)". The point estimates suggest that the joint cumulative distribution function is higher in the US than in Canada over the distribution of poor and fair health statuses, regardless of the income thresholds - despite the fact that the US marginal distribution of income dominates almost everywhere that of Canada (recall Figure 1). This is possible because Canada dominates in the lower marginal distribution of health status (recall Table 1), and that this is not offset by a greater correlation of health and income in Canada. Thus, we would conclude from these point estimates that Canada has greater joint income-health social welfare than the US distribution if the emphasis is put on those with the two least good health statuses (poor and fair).

We can also use the point estimates to see how the US-Canada ranking is sensitive to taking into account income inequality. This is done by considering the second-order estimates under the columns headed by "(2)". These estimates show that for low income thresholds average poverty gaps are greater in the US than in Canada, the more so when we focus on the least healthy individuals. The

\footnotetext{
${ }^{6}$ The cumulative income distribution function is given by $F(y)=\sum_{k=1}^{K} \phi(k) F(y ; k)$.
} 
social welfare cost of income inequality is then compounded in the US by a greater concentration of the least healthy among the poorer individuals. The dominance of Canada over the US now extends over all health statuses (up to "excellent") if $z$ (good) is no greater than $\$ 25,000$ and if $z$ (very good) and $z$ (excellent) do not exceed $\$ 15,000$. Thus, all of the social welfare functions $W(z(1), \ldots, z(K))$ in (1) that obey assumptions (2), (3), (4) and (7) will say that welfare in Canada is greater than in the US for a range of lower values of $\zeta(k) \leq z(k), k=1, \ldots, K$. This is a rather strong ranking given again the ambiguity of the ranking that could be made using only the marginal income and health distributions.

Point estimates, however, can only serve to rank the two samples. For population inferences, we must take into account sampling variability. We can proceed to statistical inference over the entire populations by verifying whether the confidence intervals shown in Table 2 contain zero. Almost all of the first-order dominance confidence intervals (those under the columns "(1)") do contain zero. We cannot therefore use the results of Theorem 1 to rank the two populations with sufficient statistical confidence. The second-order dominance confidence intervals also almost always contain zero, except for the combination of poor and fair health statuses for which Canada dominates statistically the US at all income thresholds. If therefore, we focus only and equally on those with poor and fair health statuses, and if therefore we assume that $\omega_{\text {poor }}^{(1)}(y)=\omega_{\text {fair }}^{(1)}(y), \forall y$ in (3), and that $\omega_{k}^{(1)}(y)=0$ for all higher $k$, then it is possible to say that the Canadian joint welfare distribution is better than the US one. Given the ambiguous ranking of the marginal distributions of health and income, this must again be due to the fact that the worse income distribution function is Canada (due to generally lower income levels) is compensated by a better health distribution, by a lower correlation between health and income, and by lower income inequality in Canada than in the US.

\subsection{Temporal comparisons}

We also use national health surveys to analyze trends in the joint health-income distribution during the last decade in Canada and in the US. For the US, we use the United States National Health Interview Survey (NHIS) for 1997 and 2005. For Canada, the analysis is carried out using the Canadian National Population Health Survey (NPHS) in 1996 and the Canadian Community Health Survey (CCHS) in 2005. For both countries, incomes are inflated to 2005 prices using the respective consumer price indices. 
Table 3 presents cumulative frequencies of health statuses for each country at each time period. For both the US and Canada, the distribution of health status seems to have worsened between the two surveys. Figure 2 plots the cumulative distribution functions of the 1996 and 2005 Canadian income distributions and Figure 3 plots those of the 1997 and 2005 American income distributions. The income distribution functions have improved in both countries over a wide range of possible income thresholds. Again, judging from the marginal distributions, the ranking of the two pairs of distributions jointly over income and health would therefore seem to be ambiguous.

Table 4 presents $95 \%$ confidence intervals for changes in bi-dimensional welfare in the US over the years 1997 and 2005. The sample estimates and the confidence intervals provide congruent evidence, so we focus on whether population rankings can be inferred. The confidence intervals for the differences $\Delta F(y ; k)$ and $\Delta D(y ; k)$ all contain zero for values of $k$ lower than "very good". It is only for $k$ equal to "very good" and "excellent" that 2005 dominates 1997. Thus, only if we abstract from heterogeneity in health statuses can we rank the two years and conclude that welfare has improved over time in the US. This is in part because the health distribution has deteriorated over time, but also because this deterioration has not been offset by sufficient improvements in incomes for the least healthy.

Table 5 presents $95 \%$ confidence intervals for differences in joint healthincome welfare in Canada between 1996 and 2005. Again, we focus on whether population rankings can be inferred and we thus check whether the confidence intervals contain zero. The results are statistically stronger than for the US in Table 4. Two important findings can be drawn.

Firstly, the least healthy ("poor" health status) have seen a statistically significant deterioration of their welfare. Thus, if we focus on the least healthy for the purposes of social welfare evaluation, we must conclude that 2005 is dominated by 1996. This is because the population share of the least healthy has increased over time, and because the income distribution has not sufficiently tilted in favor of the least healthy - a tilt in favor of the least healthy could have occurred either through a general improvement in mean income, a fall in the correlation between income and health status, or a fall in income inequality among the least healthy.

Secondly, if we group all of three lowest health groups into a single group, we must instead broadly conclude that 2005 dominates 1996. For first-order dominance, this is true for all $z(k)$ below $\$ 40,000$. For second-order dominance, this is true for all $z(k)$ below $\$ 50,000$. This ranking, which is rather strong, indicates that if we are willing to ignore part of the heterogeneity in lower health statuses, then we can also disregard some of the conflicting temporal evidence provided by 
the marginal income and health distributions.

Thus, if we choose not to focus only on the situation of the two least healthy groups, then we can conclude that the Canadian improvement in the income distribution has almost certainly dominated, from a joint welfare perspective, the worsening of the health distribution. If, however, we decide to focus exclusively on the situation of the group of the least healthy, then we also resolve the conflicting uni-dimensional health-income evidence, but this time we must instead conclude that joint welfare has worsened in Canada over time. In both cases, the ranking is robust over a wide class of possible income thresholds $z(k)$ and joint health-income welfare functions $W(z(1), \ldots, z(K))$, but the conclusions depend strikingly on which part of the joint health-income distribution is judged to be of greater normative importance.

\section{Conclusion}

This paper presents bi-dimensional stochastic dominance procedures that can help rank joint distributions of health and income across space and/or time. The procedures followed are more general than comparisons of health gradients since they are sensitive to changes in the entire joint distribution of health and income. Furthermore, they do not require the estimation of health equivalent incomes and are more robust to aggregation techniques than the aggregation procedures usually found in the literature.

The procedures are illustrated by ranking Canada and the US using data from the Joint Canada/United States Survey of Health. Canada is found to dominate the US over the two groups of lower health statuses in terms of the bi-dimensional distribution of health and income. Given the ambiguous ranking of the marginal distributions of health and income, this must be due to the fact that the worse income distribution function in Canada is offset partly by a better health distribution, by a lower correlation between health and income, and by lower income inequality in Canada than in the US.

The paper also uses recent national health and income data to compare welfare in Canada and the US over time. Both in the US and in Canada, the ranking over time of the uni-dimensional distributions of health and income is ambiguous. Social welfare defined jointly over income and health cannot be said to have improved in the US during the last decade in spite of the fact that the US unidimensional distribution of income did improve significantly during that period

- it is only if we abstract from heterogeneity in health statuses that we can in- 
fer an improvement in US welfare over time. This is in part because the health distribution has deteriorated over time, but also because this deterioration has not been offset by sufficient increases in incomes for the least healthy. In Canada, the temporal ranking depends strikingly on which part of the joint health-income distribution is judged to be of greater normative importance. Aggregating the three least healthy groups together, it can be inferred that the Canadian improvement in the income distribution has dominated, from a joint welfare perspective, the worsening of the health distribution. Focussing exclusively on the distribution of the group of the least healthy, it must instead be concluded that joint welfare distribution has worsened in Canada in the last decade. 


\section{A Proof of Theorem 1}

We first use (1) and integrate by parts the difference $W_{A}-W_{B}$. We find:

$$
W_{A}-W_{B}=\sum_{k=1}^{K}\left[\omega_{k}(y) \Delta F(y ; k)\right]_{0}^{\infty}-\sum_{k=1}^{K} \int_{0}^{\infty} \omega_{k}^{(1)}(y) \Delta F(y ; k) .
$$

Since $F(\infty)=1, \omega_{k}(\infty)=c, F(0)=0$ and since $\omega_{k}(0)$ is finite, then, for $W_{A}-W_{B}>0$, we have to show that

$$
\sum_{k=1}^{K} \int_{0}^{\infty} \omega_{k}^{(1)}(y) \Delta F(y ; k)<0 .
$$

Recall that $\omega_{k}^{(1)}(y)=0$ if $y>z(k)$. Combined with (2), we can then re-write (12) as:

$$
\int_{0}^{z(1)} \sum_{k=1}^{K} \omega_{k}^{(1)}(y) \Delta F(y ; k) d y<0 .
$$

The inner sum in (13) can be rewritten as:

$$
\begin{array}{r}
\sum_{k=1}^{K} \omega_{k}^{(1)}(y) \Delta F(y ; k)=\omega_{K}^{(1)}(y) \sum_{l=1}^{K} \Delta F(y ; l) \\
+\left(\omega_{K-1}^{(1)}(y)-\omega_{K}^{(1)}(y)\right) \sum_{l=1}^{K-1} \Delta F(y ; l) \\
+\ldots+\left(\omega_{1}^{(1)}(y)-\omega_{2}^{(1)}(y)\right) \Delta F(y ; 1) .
\end{array}
$$

Denoting $\omega_{K+1}^{(1)}(y)=0$, we can thus rewrite the left-hand-side of (13) as

$$
\int_{0}^{z(1)} \sum_{i=1}^{K}\left[\left(\omega_{i}^{(1)}(y)-\omega_{i+1}^{(1)}(y)\right) \sum_{k=1}^{i} \Delta F(y ; k)\right] d y
$$

with $\omega_{i}^{(1)}(y)-\omega_{i+1}^{(1)}(y) \geq 0, \forall i=1, \ldots, K$ (given condition (3)). Note also that $\omega_{i}^{(1)}(y)=\omega_{i+1}^{(1)}(y)=0$ for all $y>z(i)$ by conditions (2) and (4).

We can now establish sufficient and necessary conditions for $W_{A}-W_{B}>0$.

Sufficient condition. $\sum_{k=1}^{i} \Delta F(y ; k)<0, \forall y<z(i), \forall i=1, \ldots, K$.

Necessary condition. Suppose $\sum_{k=1}^{i} \Delta F(y ; k) \geq 0$ and $w_{k}^{(1)}(y)=w_{k+1}^{(1)}(y)$ everywhere, except for $k=i$. Then, by (17), $W_{A}-W_{B} \leq 0$, which therefore shows the necessity of $\sum_{k=1}^{i} \Delta F(y ; k)<0, s \forall y<z(i), \forall i=1, \ldots, K$. 


\section{References}

Allison, R. and J. Foster (2004). Measuring health inequality using qualitative data. Journal of Health Economics 23, 505-524.

Atkinson, A. B. and F. Bourguignon (1987). Income Distribution and Differences in Needs. New York: Macmillan.

Atkinson, T. (1992). Measuring poverty and differences in family composition. Economica 59, 1-16.

Atkinson, T. and F. Bourguignon (1982). The comparison of multi-dimensional distributions of economic status. Review of Economic Studies 49, 183-201.

Contoyannis, P. and M. Forster (1999). The distribution of health and income : a theoretical framework. Journal of Health Economics 18(5), 605-622.

Cutler, D., A. Deaton, and A. Lleras-Muney (2006). The determinants of mortality. Journal of Economic Perspective 20(3), 97-120.

Deaton, A. (2003). Health, inequality, and economic development. Journal of Economic Literature 16, 113-158.

Deaton, A. and C. Paxson (1998). Aging and inequality in income and health. American Economic Review 88, 248-253.

Humphries, K. H. and E. van Doorslaer (2000). Income-related health inequality in Canada. Social Science \& Medecine 50, 663-671.

Jenkins, S. and P. Lambert (1993). Ranking income distributions when needs differ. Review of Income and Wealth 39(4), 337-56.

Marmot, M. (2002). The influence of income on health: views of an epidemiologist. Health Affairs 21, 31-46.

O'Neill, J. E. and D. M. O'Neill (2007). Health status, health care and inequality: Canada vs the US. NBER Working Paper 13429.

van Doorslaer, E. and A. M. Jones (2003). Inequalities in self-reported health: validation of a new approach to measurement. Journal of Health Economics 22(1), 61-87.

Wagstaff, A., P. Paci, and E. van Doorslaer (1991). On the measurement of inequalities in health. Social Science \& Medicine 33, 545-557.

Wagstaff, A. and E. van Doorslaer (1994). Measuring inequalities in health in the presence of multiple-category morbidity indicators. Health Economics 3(4), 281-291. 
Wagstaff, A. and E. van Doorslaer (2000). Equity in health care finance and delivery. in : Culyer, A.J. and J.P. Newhouse, eds, Handbook of Health Economics, Elsevier, Amsterdam.

Zheng, B. (2006). Measuring health opportunity. Department of Economics University of Colorado Working Paper No 06-06. 
Figure 1: INCOME DISTRIBUTION FUNCTIONS IN CANADA AND IN THE USA

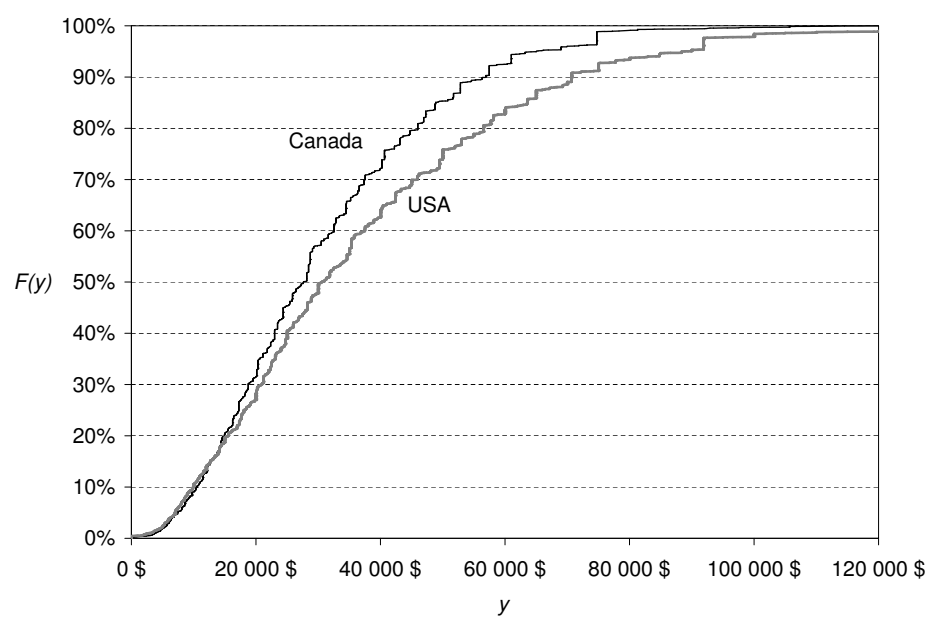

Sources: Authors' own computations using JCUSH (2003). The Canadian currency is converted into 2003 US dollars. 
Table 1: Cumulative FReQuencies of HeAlth StATUses

\begin{tabular}{|c|c|c|}
\hline Health status & $\begin{array}{c}\text { Canada } \\
(\%)\end{array}$ & $\begin{array}{l}\text { USA } \\
(\%)\end{array}$ \\
\hline Poor & 3.3 & 3.8 \\
\hline$\leq$ Fair & 11.3 & 13.6 \\
\hline$\overline{\leq}$ Good & 38.3 & 39.7 \\
\hline$\overline{\leq}$ Very good & 75.6 & 73.3 \\
\hline$\leq$ Excellent & 100.0 & 100.0 \\
\hline
\end{tabular}

Sources: Authors' own computations using JCUSH (2003). 


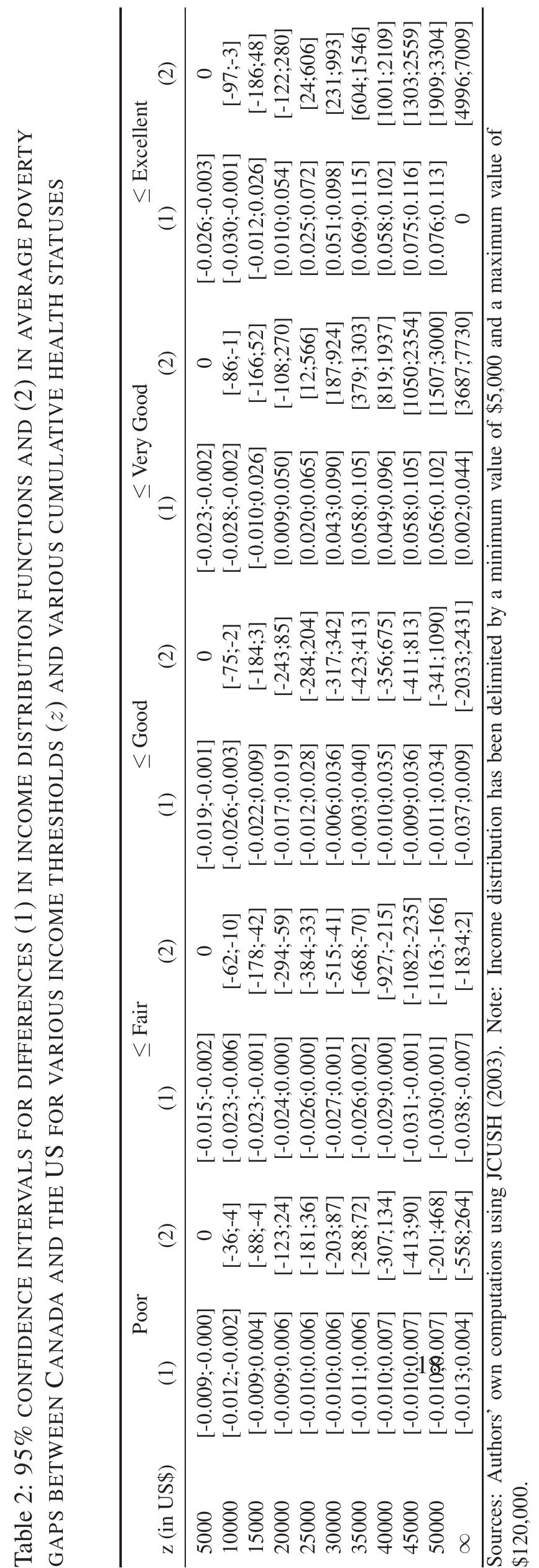


Table 3: EVOLUTION OF CUMULATIVE FREQUENCIES OF HEALTH STATUSES

\begin{tabular}{|c|c|c|c|c|}
\hline \multirow[b]{2}{*}{ Health status } & \multicolumn{2}{|c|}{ Canada } & \multicolumn{2}{|c|}{ USA } \\
\hline & $\begin{array}{c}1996 \\
(\%)\end{array}$ & $\begin{array}{c}2005 \\
(\%)\end{array}$ & $\begin{array}{c}1997 \\
(\%)\end{array}$ & $\begin{array}{c}2005 \\
(\%)\end{array}$ \\
\hline Poor & 1.8 & 2.6 & 2.2 & 2.3 \\
\hline$\leq$ Fair & 8.1 & 10.7 & 8.9 & 9.3 \\
\hline$\leq$ Good & 32.4 & 38.9 & 31.5 & 33.5 \\
\hline$\leq$ Very good & 70.0 & 77.8 & 61.7 & 64.5 \\
\hline$\leq$ Excellent & 100.0 & 100.0 & 100.0 & 100.0 \\
\hline
\end{tabular}

Sources: Authors' own computations using NPHS (1996), CCHS (2005) and NHIS (1997 and 2005). 


\section{Figure 2: Evolution of INCOME Distribution IN CANADA}

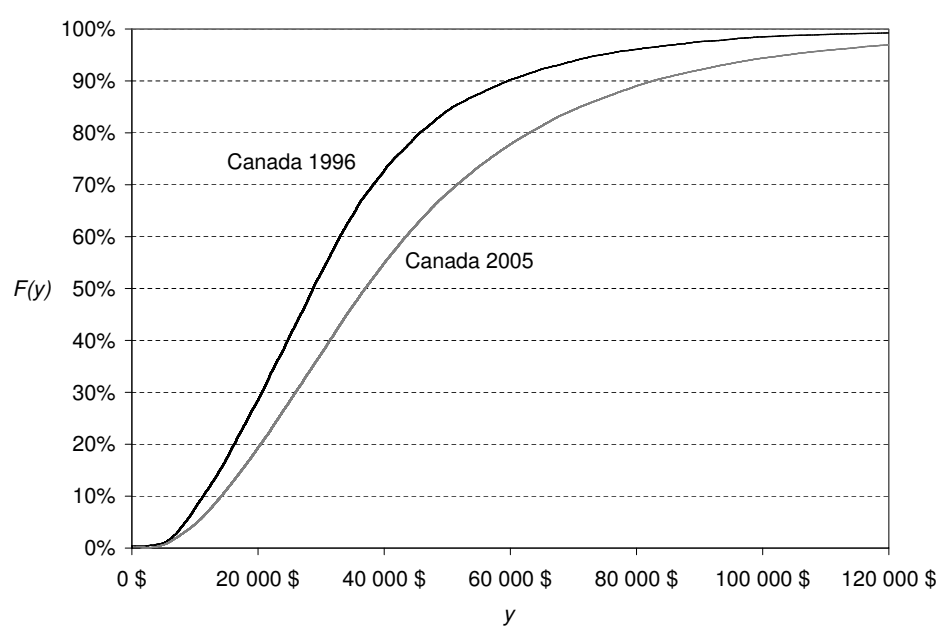

Sources: Authors' own computations using NPHS (1996) and CCHS (2005). Note: Currencies are 2005 Canadian dollars. 
Figure 3: EvOlution OF INCOME DISTRIBUTION IN THE USA

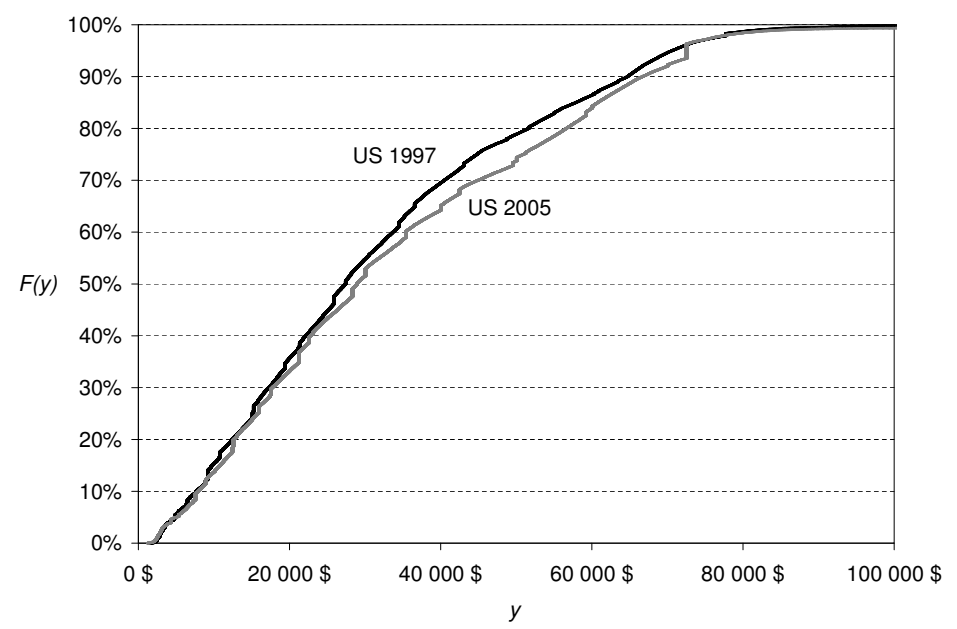

Sources: Authors' own computations using NHIS (1997 and 2005). Note: Currencies are 2005 US dollars. 


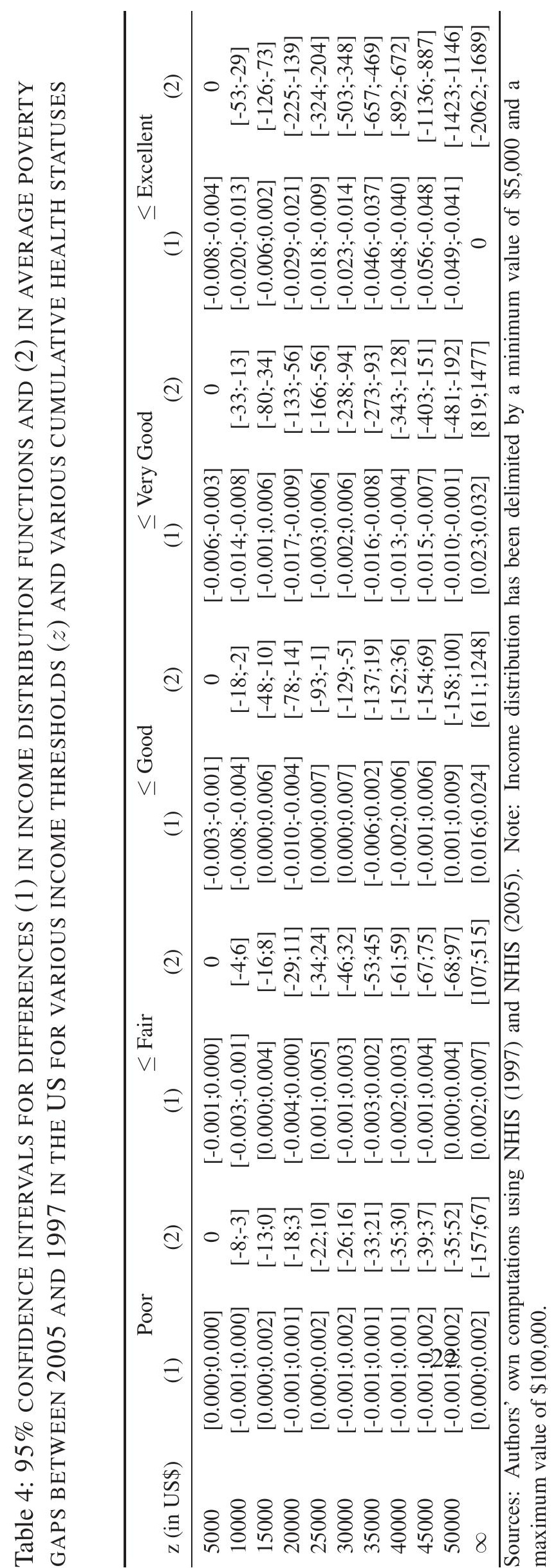




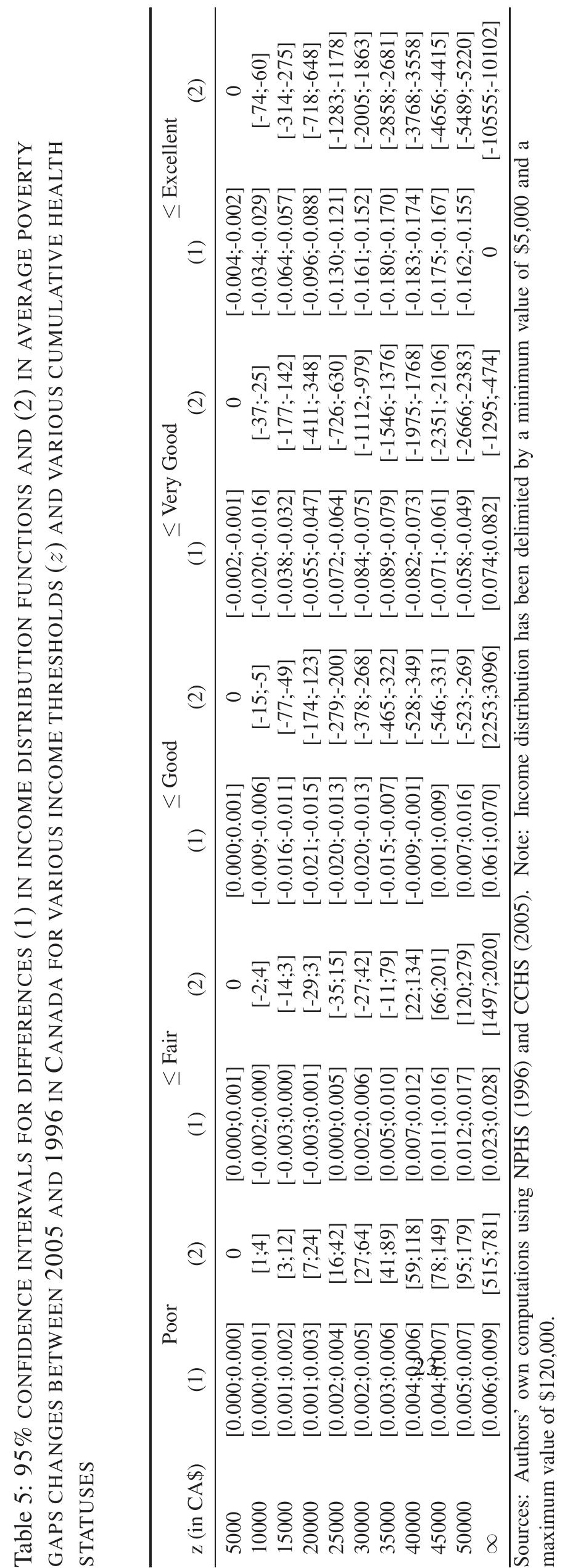

